\title{
Crisis de refugiados en Europa: conocimientos y habilidades en patología dermatológica, un instrumento clave en la cooperación internacional
}

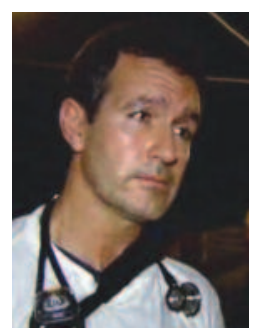

\begin{abstract}
«En realidad, es una cuestión de geografía. Todo consiste en haber nacido en este o aquel lado de la Tierra, por encima de un determinado paralelo o por debajo».
\end{abstract}

R. MONTERO Prólogo del libro de Sebastião Salgado: Sahel, el fin del camino. Médicos Sin Fronteras (MSF), 1988.
R. Ignacio Rodríguez Médico cooperante. Máster en Salud Pública Internacional.

Escuela Nacional de Sanidad.

Instituto de Salud Carlos III. Madrid.

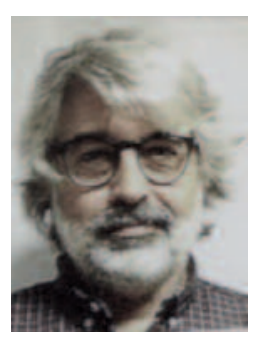

José Luis del Barrio Profesor del área de Medicina Preventiva y Salud Pública. Universidad Rey Juan Carlos. Alcorcón (Madrid).

\section{INTRODUCCIÓN}

La cooperación internacional ha evolucionado mucho en los últimos 30 años. Los protocolos y líneas de actuación sufrieron grandes modificaciones tras la crisis de los Grandes Lagos en Ruanda en 1994, así como tras el conflicto serbobosnio (1992-1995), las grandes hambrunas o la reciente epidemia de Ébola.

Las catástrofes naturales y los conflictos armados han asolado gran parte de nuestro planeta. El tsunami del sudeste asiático en 2004 o el terremoto de Haití pusieron a prueba la capacidad de respuesta de la comunidad internacional.

Las cifras de refugiados en el mundo en 2015 y 2016 son sobrecogedoras. El Alto Comisionado de Naciones Unidas para los Refugiados (ACNUR), en su informe anual Tendencias Globales, publica que 65,3 millones de per- sonas se encontraban desplazadas a finales de 2015. Esta es la primera vez que se supera el umbral de los 60 millones de personas ${ }^{1}$.

La persecución, los conflictos y la pobreza han obligado a más de un millón de personas a huir hacia Europa en 2015, el nivel de desplazamiento más grande observado en Europa occidental y central desde la década de 1990, cuando estallaron varios conflictos en la antigua Yugos lavia ${ }^{1}$.

Más de 3700 personas han perdido la vida en el mar en su intento de alcanzar las costas europeas en 2015. Los que sobreviven y logran llegar a suelo europeo, a menudo, se encuentran con más dificultades, especialmente, con la llegada del frío y las lluvias, que agravan el sufrimiento de estas personas y pueden generar más pérdidas de vidas si no se toman medidas urgentes ${ }^{2}$. 
Según el Fondo de las Naciones Unidas para la Infancia (UNICEF), en 2016, más de 350000 personas atravesaron el Mediterráneo desde el norte de África tras realizar un peligroso viaje. El $26 \%$ fueron niños refugiados y migrantes. Además, muchos llegaron solos y el frío pone en peligro la vida de miles de estos niños ${ }^{3}$.

\section{GRUPOS VULNERABLES}

Como grupos vulnerables, las mujeres y las niñas se ven afectadas a causa de su estatus en la sociedad $^{4}$.

Los menores de edad son un grupo muy vulnerable, que precisa protección y salvaguardar su estatus jurídico, además de necesitar cuidados de salud y psicológicos adaptados; también debemos prestar especial atención a las personas afectadas de alguna discapacidad y los ancianos. No hay que olvidar a las víctimas de violencia en cualquiera de sus formas, y establecer medidas de prevención.

\section{ENFERMEDADES MÁS FRECUENTES Y ESTRATEGIAS DE INTERVENCIÓN}

La Organización Mundial de la Salud (OMS), a través de su Oficina Regional para Europa, hace referencia a la existencia de medidas insuficientes de desinfección y saneamiento en muchos centros de migración que soportan flujos de personas que superan su capacidad ${ }^{5}$. Muchas enfermedades se ven agravadas por estas condiciones.

La diarrea, las infecciones respiratorias agudas y el sarampión se encuentran entre las principales causas de mortalidad en los campos de refu$\operatorname{giados}^{6}$. Según la OMS, muchos de los problemas crónicos de salud de los refugiados son, en general, similares a los que hay en la población de los países de acogida ${ }^{7}$, agravados por los efectos físicos y psicológicos de la guerra y el largo viaje. La falta de agua y saneamientos adecuados y el hacinamiento incrementan el riesgo de enfermedades transmisibles, siendo los niños especialmente susceptibles a desarrollar diarreas e infecciones respiratorias y de la $\mathrm{piel}^{7}$.

La OMS publica en otro documento que los problemas de salud incluyen lesiones por accidentes, hipotermia, quemaduras, problemas cardiovasculares, complicaciones del embarazo y el parto, diabetes e hipertensión arterial $^{5}$.

El ahogamiento es una causa muy importante de mortalidad entre los que cruzan el Mediterráneo.

La ruta que deben afrontar los flujos de refugiados por tierra y mar es muy peligrosa; esta es una de las razones por las que numerosas organizaciones humanitarias, como por ejemplo Médicos sin Fronteras (MSF), reclaman las Vías Seguras para los refugiados ${ }^{8}$.

En 2016, MSF reanuda las actividades de búsqueda y rescate en el Mediterráneo. En 2015, varios equipos llevaron a cabo estas labores, asistiendo a más de 23000 personas. Las principales enfermedades observadas entre los rescatados fueron hipotermias, deshidratación, enfermedades de la piel y quemaduras causadas por combustible. En total, en 2015, los equipos de la organización trataron a más de 100000 personas en el Mediterráneo, el mar Egeo, Grecia, Italia y los Balcanes ${ }^{9}$.

Decenas de migrantes y refugiados, alerta Save the Children en enero de 2017, corren el riesgo de morir congelados en Europa, tras las nevadas y bajas temperaturas que han golpeado a Grecia y a los Balcanes en los últimos días ${ }^{10}$.

Hasta el momento, más de 40 refugiados han muerto por el frío. En los campos de refugiados de la zona, los niños y bebés son los más vulnerables a sufrir hipotermia, al no tener forma de calentarse. Muchos viven en campamentos mal equipados, en edificios abandonados o en calles llenas de nieve ${ }^{10}$.

En Belgrado, donde las temperaturas han llegado hasta los 10 grados bajo cero, cientos de niños no acompañados, algunos de tan solo 10 y 11 años de edad, están entre los 1200 refugiados y migrantes que duermen en el suelo de edificios abandonados y almacenes. Estos edificios no tienen ventanas, camas, agua ni $\operatorname{aseos}^{10}$. 
MSF, desde hace tiempo, ha establecido estrategias y prioridades en intervenciones de acción humanitaria que implican atención sanitaria a refugiados ${ }^{11}$; estas prioridades son una herramienta muy útil para la comunidad humanitaria. Entre ellas, destacan: la vacunación (se hace especial énfasis en la vacunación contra el sarampión) y el acceso a agua y saneamientos, a un refugio y a cuidados de salud.

La OMS y UNICEF, también en caso de población refugiada, establecen la vacunación como prioridad y, en el caso de la crisis migratoria de refugiados en Europa, reconocen problemas en la planificación de estrategias, debido a la magnitud y velocidad del flujo migratorio, y consideran que el sarampión, la parotiditis y la rubeola deberían ser prioritarios ${ }^{7}$.

Establecer estrategias de intervención en las crisis humanitarias es un objetivo prioritario desde hace tiempo por parte de las organizaciones humanitarias. El Proyecto Esfera fue creado en 1997. Lo componen representantes de 18 de organizaciones internacionales, como, por ejemplo, Caritas Internacional, Save the Children, Care, Cruz Roja y Media Luna Roja, etc. El proyecto establece unas normas mínimas y principios comunes para la respuesta humanitaria ${ }^{12}$.

\section{PROBLEMAS DE SALUD RELACIONADOS CON LA DERMATOLOGÍA}

Es evidente que la procedencia de países en conflicto, las características de las rutas y el hacinamiento en los refugios pueden producir lesiones y enfermedades de la piel.

Estudios realizados en Turquía, lugar donde se encuentra un alto número de refugiados, evidencian haber encontrado problemas cutáneos de diversa etiología: erupciones, sarna ${ }^{13}$ y leishmaniosis cutánea.

A causa de la guerra en Siria, Turquía ha recibido en torno a tres millones de refugiados ${ }^{14}$. Un total de 567 personas precisaron ingreso hospita- lario con la sospecha de leishmaniosis cutáneas, de los cuales, $263(46,4 \%)$ resultaron positivos en el examen parasitológico ${ }^{14}$.

En un estudio realizado en Bruselas en 2015 por Medecins du Monde (MDM) en un centro de refugiados, el $9 \%$ de los refugiados presentaban afecciones dermatológicas ${ }^{15}$.

Wollina et al. realizan un valioso estudio en un campo temporal de refugiados en Dresde (Alemania) durante 10 semanas en 2015; el diagnóstico más común fue sarna; en segundo lugar, dermatitis eccematosa e impétigo; con menor frecuencia, trataron otros problemas como varicela, foliculitis, abscesos y urticaria. Refieren que, sorprendentemente, no observaron leishmaniosis cutánea entre los solicitantes de asilo atendidos, aunque la mayor parte provinieran de Siria ${ }^{16}$.

En todos los contextos, debemos prestar atención especial a las lesiones relacionadas con enfermedades de transmisión sexual.

Aparte de las aportaciones anteriores, es muy valiosa la contribución de la European Academy of Dermatology and Venereology (EADV), que emitió un comunicado de prensa sobre la dermatología y los refugiados en el 13. ${ }^{\text {er }}$ Simposio, que tuvo lugar en Grecia en 2016. En dicho comunicado, se abordó el argumento de como las enfermedades de la piel pueden crear un impacto en los refugiados. Entre los puntos más relevantes de dicho comunicado, se encuentran los siguientes.

«Es tiempo de hablar sobre la crisis de refugiados en Europa en relación con nuestra especialidad». También se puede leer más adelante que «las enfermedades de la piel son frecuentes en los países menos desarrollados y en las comunidades más desfavorecidas, produciendo incomodidad, desfiguraciones e, incluso, marginación. La falta de higiene y las condiciones de hacinamiento en las cuales los refugiados viven favorecen el desarrollo y la difusión de enfermedades de la piel. Sin embargo, muchas de estas enfermedades tienen fácil solución» ${ }^{17-19}$.

«Es nuestra responsabilidad —continúa el comunicado de prensa de la EADV- propor- 
Rodríguez RI et al. Crisis de refugiados en Europa: conocimientos y habilidades en patología dermatológica, un instrumento clave en la cooperación internacional

cionar cuidados y también formar a los trabajadores humanitarios que desarrollan su labor en campos para que diagnostiquen apropiadamente y usen los tratamientos más simples en relación coste-efectividad y las medidas de prevención disponibles» ${ }^{7-19}$. «Los refugiados e inmigrantes frecuentemente proceden de comunidades afectadas por la guerra o las crisis económicas y han realizado largos y exhaustivos viajes que incrementan sus riesgos de enfermar. A su llegada, las enfermedades de la piel más frecuentes están relacionadas con la falta de higiene y la vida en condiciones de hacinamiento; entre dichas enfermedades, nos encontramos sarna, pediculosis y enfermedades bacterianas de la piel. Las quemaduras y otras lesiones traumáticas de la piel sobreinfectadas son también frecuentes, así como las dermatitis causadas por la exposición solar y el agua del mar», refirió una de las dermatólogas del Hospital Mater Dei de Malta. También se hizo referencia por parte de dermatólogos italianos a la «necesidad de reconocer enfermedades que no estamos habituados a ver en nuestro contexto como la leishmaniosis cutánea y la tuberculosis cutánea»17-19.

Por último, se destaca en el comunicado de la EADV: «Hay informes que señalan que la tortura ha sido común en muchos países del mundo en los últimos años, y los refugiados frecuentemente proceden de grupos vulnerables. Los que han sobrevivido a la tortura no hablan de sus experiencias, incluso, al personal médico. Se puede encontrar en su piel signos físicos que deberían ser reconocibles. Incluso cuando la piel ha cicatrizado, los supervivientes a la tortura pueden presentar síndrome de estrés postraumático, que puede provocar efectos devastadores como ansiedad, depresión, aislamiento, déficits de memoria y atención, pesadillas y muchos más síntomas. Muchas de las secuelas neurológicas y traumatológicas pueden tener su origen en la tortura, por lo que es importante que los profesionales sanitarios deriven a estas víctimas para que reciban un apropiado apoyo médico y psicológico» ${ }^{17-19}$.

\section{CONCLUSIONES}

A través de prioridades claramente definidas y siguiendo protocolos adaptados a diferentes situaciones, las organizaciones humanitarias se enfrentan a nuevos retos y dificultades para atender a la población desplazada o refugiada en sus asentamientos. Existen problemas añadidos como son los movimientos migratorios por rutas peligrosas, por el mar Mediterráneo y, por tierra, a través de los Balcanes.

La ruta del mar Mediterráneo implica un riesgo muy elevado, al navegar en embarcaciones en mal estado, por lo que las organizaciones humanitarias, así como los países europeos, se han visto obligados a implementar estrategias y utilizar recursos para realizar tareas de salvamento. Pese a todo ello, el esfuerzo resulta insuficiente ante el grandísimo flujo migratorio.

El conocimiento y la formación en salud pública, cuidados básicos de salud desde el enfoque de atención primaria, formación en especialidades relacionadas con la medicina y cirugía de guerra, así como en psiquiatría, psicología y pediatría resultan claves para prestar una asistencia de calidad. Es fundamental el personal con conocimientos en operaciones de salvamento marítimo.

Las causas más frecuentes de mortalidad en campos de refugiados están relacionadas con las infecciones respiratorias, el sarampión y las diarreas. Como hemos visto, dependiendo de la ruta, en esta crisis de refugiados, hay otras causas de mortalidad como, por ejemplo, el ahogamiento y la hipotermia.

Debido al hacinamiento y las condiciones de insalubridad, la patología dermatológica es un problema frecuente, por lo que es entendible que el conocimiento y manejo de estas enfermedades por parte del personal sanitario que atiende a estos refugiados sea también un instrumento de gran utilidad, en la línea de lo expresado en el comunicado de prensa del 13. ${ }^{\text {er }}$ Simposio de Dermatología y Venereología de la EADV.

Son frecuentes, entre otras afecciones cutáneas, la sarna, la pediculosis, las dermatitis causadas por 
la exposición solar y el agua del mar, las lesiones de la piel sobreinfectadas y las quemaduras y, en determinados centros, se ha hallado leishmaniosis cutánea entre los refugiados procedentes de Siria.

El drama humano continúa en las fronteras y más allá de ellas. La presencia de personal sanitario preparado representa una esperanza para quienes recorren las rutas migratorias.

Debemos prestar especial atención a todos los grupos vulnerables, sobre todo, a los niños, que, en muchas ocasiones, llegan solos, precisando no solo atención sanitaria y psicológica, sino también protección.

\section{BIBLIOGRAFÍA}

1. UNHCR/ACNUR. Tendencias Globales sobre refugiados y otras personas de interés del ACNUR. Disponible en: http://www.acnur.org/recursos/estadisticas/

2. UNHCR/ACNUR. Emergencia en Europa. Disponible en: http://acnur.es/emergencia-en-europa

3. UNICEF. Niños refugiados y migrantes en Europa. Disponible en: https://www.unicef.es/causas/emergencias/refugiadosmigrantes-europa

4. UN High Commissioner for Refugee (UNHCR). UNHCR Handbook for the Protection of Women and Girls. 1. ${ }^{\mathrm{a}}$ ed. Ginebra: UNHCR; 2008. Disponible en: http://www.unhcr.org/ protection/women/47cfa9fe2/unhcr-handbook-protectionwomen-girls-first-edition-complete-publication.html

5. World Health Organization Regional Office for Europe. Refugee Crisis. Situation update 3. Copenhague: World Health Organization Regional Office for Europe; 2016. Disponible en: http://www.euro.who.int/_data/assets/pdf_file/0016/ 305503/Refugee-Crisis-situation-update-report-n3.pdf

6. Spotlights on health and rights. Key topics in the Heilbrunn Department of Population and Family Health. Basic facts. Disponible en: http://healthandrights.ccnmtl.columbia.edu/ forced_migration/basic_facts.html

7. World Health Organization Regional Office for Europe. WHOUNHCR-UNICEF joint technical guidance: general principles of vaccination of refugees, asylum-seekers and migrants in the WHO European Region. World Health Organization Regional Office for Europe. News. 23-11-2015. Disponible en: http://www.euro.who.int/en/health-topics/disease-prevention/vaccines-and-immunization/news/news/2015/11/who,unicef-and-unhcr-call-for-equitable-access-to-vaccines-forrefugees-and-migrants/who-unhcr-unicef-joint-technical-guidanc e-general-principles-of-vaccination-of-refugees,-asylum-seekers-and-migrants-in-the-who-european-region
8. Médicos Sin Fronteras. Vías Seguras. MSF. Actualidad. 2301-2017. Disponible en: https://www.msf.es/actualidad/estapasando/vias-seguras

9. Médicos Sin Fronteras. Mediterráneo: "aquí estamos otra vez, denunciando las consecuencias de la falta de vías seguras para llegar a Europa". MSF. Actualidad. Noticias. 09-052016. Disponible en: https://www.msf.es/actualidad/mediterraneo/mediterraneo-aqui-estamos-otra-vez-denunciando-las-c onsecuencias-la-falta

10. Save the Children. Save the Children denuncia que la Unión Europea está abandonando en el frío y la nieve a miles de niños refugiados. Save the Children. 11-01-2017. Disponible en: https://www.savethechildren.es/notasprensa/save-children-denuncia-que-la-union-europea-esta-abandonando-enel-frio-y-la-nieve-miles

11. Depoortere E, Brown V. Rapid health assessment of refugee or displaced population. 3. ${ }^{\mathrm{a}}$ ed. París: Epicentre Epidemiology/ Médecins Sans Frontières; 2006:11. Disponible en: http://refbooks.msf.org/msf_docs/en/rapid_health/rapid_health_en.pdf

12. El Proyecto Esfera. Cartas Humanitarias y normas mínimas para la respuesta humanitaria. El Proyecto Esfera en breve. Disponible en: http://www.sphereproject.org/sphere/es/acerca/

13. World Health Organization. Frequently asked questions on migration and health:1-4. WHO. 2017. Disponible en: http://www.who.int/features/qa/88/en/

14. Özkeklikçi A, Karakuş M, Özbel Y, Töz S. The new situation of cutaneous leishmaniasis after Syrian civil war in Gaziantep city, Southeastern region of Turkey. Acta Trop. 2017;166:358. Disponible en: http://www.sciencedirect.com/science/ article/pii/S0001706X16305484

15. van Berlaer G, Bohle Carbonell F, Manantsoa S, de Béthune $X$, Buyl R, Debacker M, et al. A refugee camp in the center of Europe: clinical characteristics of asylum seekers arriving in Brussels. BMJ Open. 2016;6(11):e013963. Disponible en: http://bmjopen.bmj.com/content/6/11/e013963.long

16. Wollina U, Gaber B, Mansour R, Langner D, Hansel G, Koch A. Dermatologic challenges of health care for displaced people. Lessons from a German emergency refugee camp. Our Dermatol Online. 2016;7(2):136-38. Disponible en: http://www.odermatol.com/odermatology/20162/2.Dermatolo gic-WollinaU.pdf

17. Dermcity. Refugee crisis: skin diseases commonly associated with refugees, migrants. Dermcity. 01-06-2016. Disponible en: http://www.derm.city/single-post/2016/06/01/Refugeecrisis-skin-diseases-commonly-associated-with-refugees-migrants

18. EADV European Academy of Dermatology and Venereology 13th EADV Spring Symposium 19-22 May 2016 Athens, Greece. Prominent dermatologists and venereologists discuss refugee crisis at EADV Symposium in Athens, Greece. EADV. Press releases. 23-06-2016. Disponible en: http://eadvathens2016.org/press/

19. EuroActiv Press Release. Prominent dermatologists and venereologists discuss refugee crisis at EADV Symposium in Athens, Greece. EADV. 23-05-2016. Disponible en: http://pr.euractiv.com/pr/prominent-dermatologists-and-venereologists-discuss-refugee-crisis-eadv-symposium-athens-greece 